\title{
LA PALEODIVERSIFICACIÓN: ALGUNAS DIFICULTADES PARA SU ESTUDIO
}

\author{
Mireille GAYET y Claude BABIN
}

Univ. Claude Bernard-Lyon I y CNRS, UFR Sciences de la Terre, 27 bd du 11 novembre, 69622 Villeurbanne Cedex, France.

Gayet, M. y Babin, C. 2001. La paleodiversificación: algunas dificultades para su estudio. [Palaeodiversification: Some problems for the study.] Revista Española de Paleontología, n. ${ }^{\circ}$ extraordinario, 117-123. ISSN 02136937.

\begin{abstract}
Two examples taken from invertebrates on the one hand (mollusc bivalves) and vertebrates (Osteichthyes, Cladistia Polypteriformes), on the other, occurring in strong different palaeoenvironments (marine versus freshwater) and which diversified at very distinct times (Lower-Middle Ordovician versus Upper Cretaceous), allow to question about the research of the inductive factors and the understanding of the modes of palaeodiversification. These questions are when? where? and how? the palaeodiversifications occurred. Strong difficulties arise to establish the impact of the palaeoenvironments and the obviousness of the key innovations. The sudden appearance of these two radiations does not fully agree with the Darwin's gradualist pattern.
\end{abstract}

Keywords: Molluscs, bivalves, fish, polypteriforms, Lower Ordovician, Upper Cretaceous, evolution.

\section{RESUMEN}

Dos ejemplos muy diferentes, invertebrados (moluscos bivalvos) y vertebrados (Osteichthyes, Cladistia Polypteriformes), de ambientes muy distintos (marinos y de aguas dulces) y que se diversificaron en momentos muy distantes (Ordovícico Inferior-Medio y Cretácico Superior) permiten plantear algunos de los problemas encontrados en la detección de los factores inductores y en la comprensión de las modalidades de paleodiversificación. Los interrogantes son ¿cuándo, dónde y cómo se han producido las paleodiversificaciones? Establecer el papel de los paleoambientes y evidenciar las innovaciones clave constituyen dificultades importantes. El carácter al parecer súbito de estas radiaciones no concuerda totalmente con el gradualismo del modelo darwiniano clásico.

Palabras clave: Moluscos, bivalvos, peces, polipteriformes, Ordovícico Inferior, Cretácico Superior, evolución.

\section{INTRODUCCIÓN}

Desde hace pocos años, el estudio de la biodiversidad (la palabra ha conocido un empleo explosivo en la literatura científica desde 1988, ver Harper y Hawksworth, 1994) ha cobrado un gran auge, extrapolable también al terreno de la Paleontología. Esta disciplina aporta numerosas investigaciones sobre los procesos y la historia evolutiva de la que surgieron los organismos actuales, centrados en el campo de la paleobiodiversificación y las extinciones habidas en el transcurso del Fanerozoico.

A decir verdad, hace mucho tiempo que la paleontología mostró que la evolución de la vida fue, numerosas veces, marcada por importantes radiaciones más o menos abruptas. Sin embargo, de la mayor parte de los trabajos presentados a un reciente simposio celebrado en Lyon (Francia), dedicado al estudio de la paleodiversificación, quedó patente la dificultad de aportar una visión clara del mecanismo por el que interaccionan los factores intrínsecos y los agentes externos o medioambientales.

Entre las numerosas interrogantes que se plantean están las siguientes: ¿qué fiabilidad tiene el registro fósil en el estudio de estos fenómenos?; ¿se puede llegar a fijar en el tiempo y en el espacio la cuna de un determinado grupo biológico?; ¿pueden llegarse a conocer las innovaciones clave que facilitaron eventualmente su diversificación? En resumen, las interrogantes son ¿cuándo, dónde y cómo se han producido las paleodiversificaciones?

En el presente trabajo pasaremos revista a algunos problemas a través del examen de dos grupos bien distintos, invertebrados y vertebrados, de ambientes 


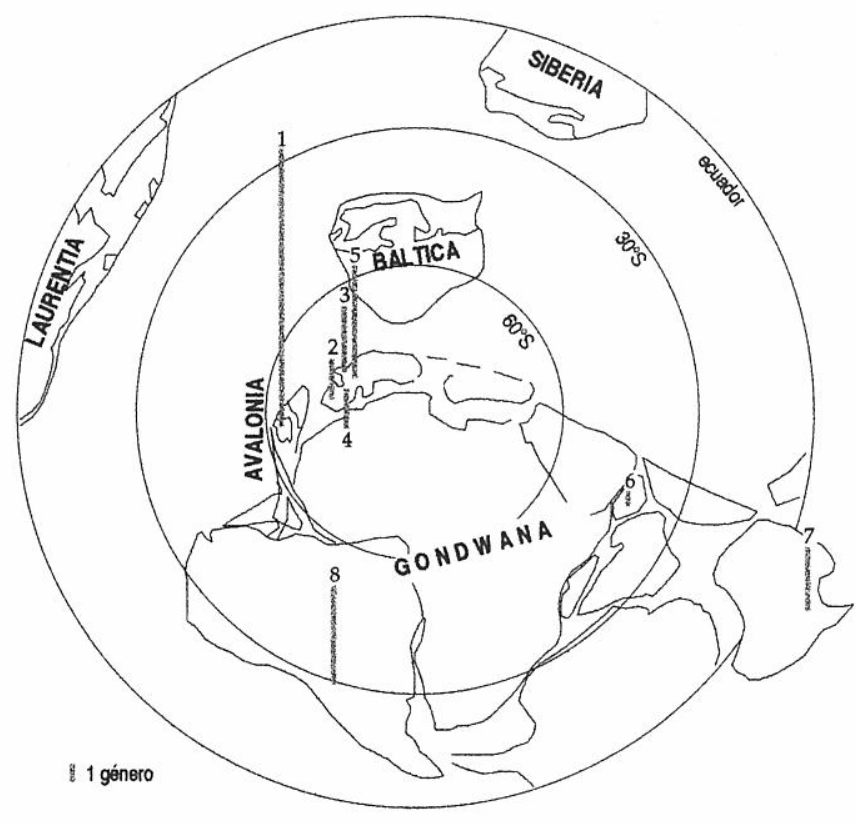

Figura 1. Distribución conocida actualmente de los bivalvos durante su diversificación en el Ordovícico Inferior (Tremadoc superior-Arenig); está localizada en las plataformas gondwánicas sin preferencia latitudinal. 1: Gales; 2, Península Ibérica; 3, Macizo Armoricano; 4, Marruecos; 5, Montagne Noire; 6, Afganistán; 7, Australia; 8, Argentina.

Actually known distribution of the bivalves during their diversification in the Early Ordovician (Late Tremadoc-Arenig); they are localised on the Gondwanian shelves without latitudinal preference. 1, Wales; 2, Iberian peninsula; 3, Armorican Massif; 4, Morocco; 5, Montagne Noire; 6, Afghanistan; 7, Australia; 8, Argentina.

diferentes y de momentos muy distantes: los moluscos bivalvos marinos del Ordovícico, y los peces polipteriformes de aguas dulces del Cretácico.

\section{MOLUSCOS BIVALVOS}

\section{EL REGISTRO FÓSIL}

Los moluscos bivalvos inician su registro muy discretamente a partir del Cámbrico Inferior, donde aparecen algunos pequeños y raros géneros, como Fordilla, bastante cosmopolita, y Pojetaia, conocido solamente en Gondwana. Durante el Cámbrico Medio, junto a Pojetaia, se conocen los nuevos géneros Tuarangia (Gondwana y Báltica), Camya (Báltica, HinzSchallreuter, 1995) y Arhouriella (Gondwana, Geyer y Strend, 1998). No está esclarecido el modo de vida de estos bivalvos minúsculos que podrían haber sido elementos de una meiofauna.

Tras una ausencia de registro fosilífero del grupo, que abarca casi todo el Cámbrico Superior así como gran parte del Tremadoc, la clase experimenta una amplia y rápida diversificación durante el Arenig. El fenómeno puede estar vinculado con el amplio desarrollo de una sedimentación eminentemente siliciclástica en las plataformas circundantes a Gondwana y a la placa contigua de Avalonia, si bien carece de un claro patrón paleolatitudinal (Babin, 1993, 1995). Ya en el Arenig Inferior aparecen representados seis de los siete órdenes de esta clase (Cope, 1996), con neto predominio de los bivalvos endobentónicos y semi-endobentónicos. Ambos alcanzan gran desarrollo durante el Ordovícico Medio (Llanvirn) en las plataformas gondwánicas, y algunos bivalvos migran esporádicamente a Báltica (Babinka) y Laurentia (Tironucula). Sin embargo, durante el Ordovícico Superior (Caradoc y Ashgill), la situación se invierte y los bivalvos declinan en todo Gondwana, aunque en Báltica y Laurentia se produce una importante diversificación de las formas epibentónicas, que llegan a colonizar los nuevos ambientes surgidos en plataformas carbonáticas. No obstante, seguimos sin conocer por qué los bivalvos se establecieron tardíamente en Báltica y Laurentia (Cope y Babin, 1999).

Permanece el problema de su abrupta diversificación en el Arenig; la casi ausencia de bivalvos durante el Cámbrico Superior y el Tremadoc podría resultar de fenómenos geodinámicos (subducciones), tafonómicos o de investigaciones insuficientes, entonces sería sólo un artificio el aspecto rápido de la radiación. Sin embargo, tenemos numerosos fósiles de otros grupos diversos de animales bentónicos durante el mismo período y además sabemos que el Ordovícico fue un momento de gran diversificación para diferentes grupos. Así, los datos que se conocen actualmente parecen concordar con el carácter amplio y bastante súbito de la paleodiversificación arenigiense de los bivalvos. ¿Qué causas pueden invocarse para el fenómeno?

\section{FACTORES EXTERNOS}

Tampoco están definidas las pautas que indujeron o favorecieron la primera diversificación de los bivalvos; se puede suponer que el sustrato clástico resultó un factor favorable para la nutrición, y que en el ecoespacio endobentónico en el que proliferaron carecían también de competidores. La temperatura o el control paleolatitudinal, por el contrario, no parece haber jugado un papel determinante en este proceso temprano de diversificación, puesto que los bivalvos arenigienses se distribuyen en diferentes partes de Gondwana: Europa, América del Sur y Australia, que se extendían desde paleolatitudes elevadas a otras paleoecuatoriales (Fig. 1).

\section{A LA BUSCA DE INNOVACIONES CLAVE}

En el terreno de las innovaciones, la adquisición de las branquias de tipo filibranquio, que facilitan la nutrición, podría haber constituído una clave decisiva, si bien se hace necesario buscar evidencias de cuándo ésta pudo llevarse a cabo (Cope, 1995, 1997). En ese sentido, resulta evidente que con las conchas como únicos restos fosilizados ( en su mayoría conservadas como moldes internos y externos en la roca!), sólo se pueden presentar hipótesis a propósito de tal adquisición. Cope propuso que la abertura de la concha podría estar relacionada con 

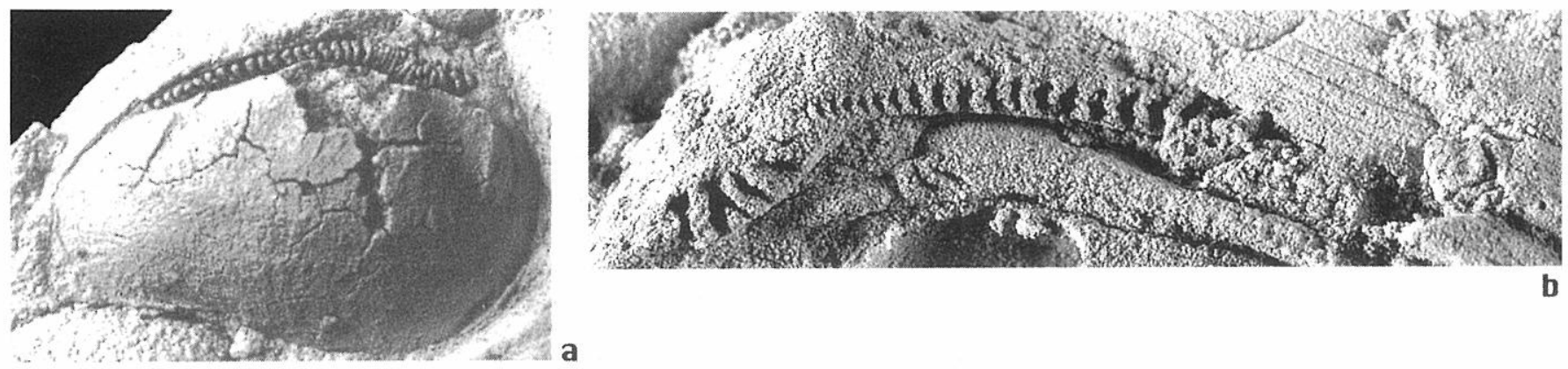

Figura 2. Cómo detectar una innovación no fosilizable: según Cope (1995), el hábito filibranquio, no fosilizable, en los moluscos bivalvos se puede relacionar con características de la dentición, fosilizada; dos paleotaxodontos del Ordovícico Medio de España (Babin y Gutiérrez-Marco, 1991), a, Myoplusia bilunata perdentata (Barrande) y b, Cardiolaria beirensis (Sharpe); el primero, con dientes apretados, no podía abrir mucho la concha, era un protobranquio clásico; el segundo, con un pequeño espacio entre las dos series de dientes, tenía la posibilidad de abrir más las valvas y podría ser un filibranquio.

How to detect a no fossilisable innovation; regarding Cope (1995), the filibranch gills, a no fossilisable character, can be linked to the peculiar dentition characters which are fossilised. Two palaeotaxodonts from the Spanish middle Ordovician (Babin and Gutiérrez Marco, 1991), a, Myoplusia bilunata perdentata (Barrande) and, b, Cardiolaria beirensis (Sharpe); the former with close teeth could not open widely the valves, it has been a regular protobranch; the later with short space between the two teeth series could open more widely the valves, and could has been a filibranch.

el tipo de branquias. El autor hace notar que la dentición clásica de los paleotaxodontos, con numerosos dientes apretados (Fig. 2a), permite sólo una abertura muy pequeña de la concha; al contrario, una dentición como la de Cardiolaria, con un espacio entre las dos series de dientes debajo del umbo (Fig. 2b), facilitaría una abertura más grande y la circulación de una corriente mayor de agua. Así, Cardiolaria podría haber tenido una dentición de tipo paleotaxodonto y branquias de tipo filibranquio.

Todo esto constituye un buen ejemplo de que, a pesar de los progresos recientes en la investigación de los primitivos bivalvos, el problema de la cuna inicial queda oscuro y se carece aún de una interpretación completamente satisfactoria para su diversificación durante el Ordovícico.

\section{PECES}

\section{REGISTRO FÓSIL}

Los peces actinopterigios ofrecen diferentes modos de "primera" aparición. Un buen ejemplo de ello son los polipteriformes (Actinopterygii, Cladistia), un grupo de peces ganoideos con algunas diversificaciones en el transcurso de su evolución. De ellos subsisten en la actualidad dos géneros, habitantes de las aguas dulces africanas, Polypterus y Calamoichthys. Polypterus es un pez habitante de las aguas dulces "primario", es decir estrictamente intolerante a las aguas marinas (Myers, 1938, 1949). El muy especializado Calamoichthys, que puede vivir en estuario, es un pez habitante de las aguas dulces "secundario". Los polipteriformes son sin duda un grupo monofilético (Gardiner, 1984). Este grupo de peces experimenta una aparición súbita y explosiva en el Cenomaniense de tres localidades africanas: en el Sudán con siete géneros y catorce especies (Werner, 1994;
Werner y Gayet, 1997), en Egipto con dos géneros y dos especies (Schaal, 1984) y en Marruecos con tres géneros y tres especies (Sereno et al., 1996; Dutheil, 1999). Así, las faunas cenomanienses caracterizadas reúnen ocho géneros y diecisiete especies distintas (Gayet et al., en prensa).

En África, el equilibrio aparición/extinción debió permanecer casi estabilizado durante todo el Cretácico Superior, cuanto menos hasta el Santoniense, al que corresponde el yacimiento del Níger que contiene seis géneros y doce especies (Gayet y Meunier, 1996).

En América del Sur, dos géneros de polipteriformes, Dajetella y Latinopollia, diferentes de los africanos, que agrupan por lo menos dos o tres especies (Gayet y Meunier, 1991, 1992, 1998; Meunier y Gayet, 1996), han sido descubiertos en los niveles maastrichtienses de Bolivia y de Brasil. En Bolivia subsisten hasta el Paleoceno terminal y después desaparecen definitivamente del continente. No obstante, los estudios en curso permitirán ampliar, muy probablemente, el número de taxones sudamericanos.

A excepción de Polypterus (Fig. 3a), todos los demás géneros africanos de polipteriformes desaparecen durante el Cretácico superior; en cambio, el ya citado Polypterus, reconocido en algunos pisos, se perpetúa hasta hoy. Con catorce especies y subespecies actuales, dicho género manifiesta una nueva y reciente diversificación en el presente, que coincide a su vez con la aparición de un nuevo género, Calamoichthys, conocido a través de una única especie. Si descontamos la breve presencia (Maastrichtiense a Paleoceno) de los polipteriformes en América del Sur, la historia evolutiva y la abundancia del grupo están limitadas claramente al continente africano.

\section{IDENTIFICACIÓN TAXONÓMICA}

Los polipteriformes son unos peces muy particulares 


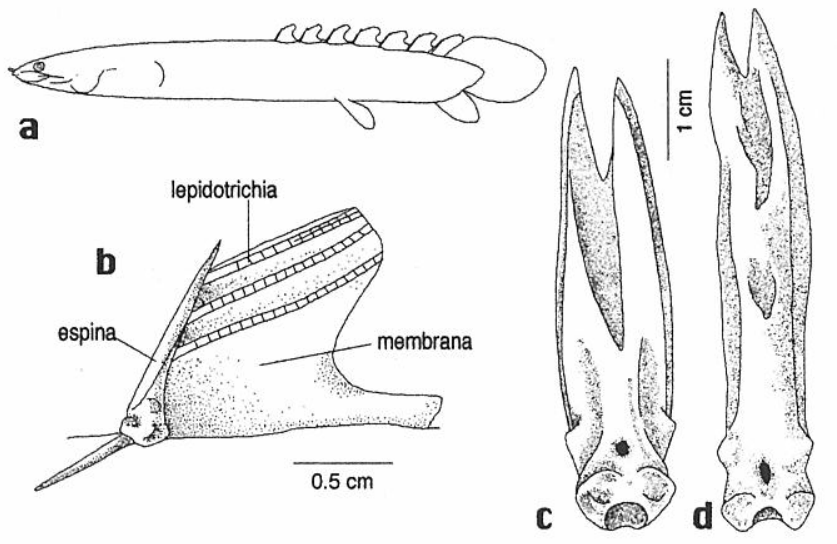

Figura 3. Polipteriformes. a, vista general de Polypterus senegalus (actual); b, pínula de la aleta dorsal del mismo; c-d, espinas de pínulas de Calamoichtys (c), y de Polypterus ornatipinnis (d).

Polypteriformes. a. general view of Polypterus senegalus (Recent); $\boldsymbol{b}$. finlet of the dorsal fin of the same genus; $c-d$, spines of finlets of Calamoichtys (c), and Polypterus ornatipinnis $(d)$.

que se caracterizan, entre otros elementos, por la posesión de una aleta dorsal discontinua, formada por un número variable de pequeñas aletas llamadas pínulas, cada una de las cuales se encuentra precedida por una espina (Fig. 3bd). El número de pínulas y la morfología de las espinas constituyen los caracteres diagnósticos esenciales de las distintas especies (Gayet et al., 1997). Además, sus vértebras y sus escamas ganoideas poseen características muy particulares, en su morfología, las primeras, y en su histología, las segundas. Así se pueden reconocer los polipteriformes con una sola escama o una sola vértebra, diferenciándose los géneros y especies en función de las pínulas. Tanto las escamas como las espinas fosilizan con facilidad por estar cubiertas de ganoina, resultando a menudo los únicos elementos conservados.

\section{ANTECESOR SUPUESTO}

Ninguno de los taxones caracterizados en los yacimientos más antiguos permitiría presagiar tal tipo de morfología y tan súbita diversificación del grupo; por el momento no se poseen evidencias de formas anatómicamente intermedias en otro taxón vinculado filogenéticamente con los polipteriformes.

\section{DINÁMICA DE LA DIVERSIFICACIÓN}

Los polipteriformes mostraron dos picos de diversificación (africanos) o acaso tres (africanos y suramericano), un potencial de diversificación del que carecieron, por ejemplo, los lepisosteiformes (Fig. 4). Estos últimos son igualmente actinopterigios con escamas ganoideas, que surgieron también en África durante el Albiense, y que llegan a coexistir en algunos yacimientos con los polipteriformes. Los lepisosteiformes tuvieron una amplia extensión geográfica dentro de cinco continentes pero sin ninguna radiación explosiva y siempre con un pequeño número de taxones (poseen únicamente dos géneros y siete especies actuales,

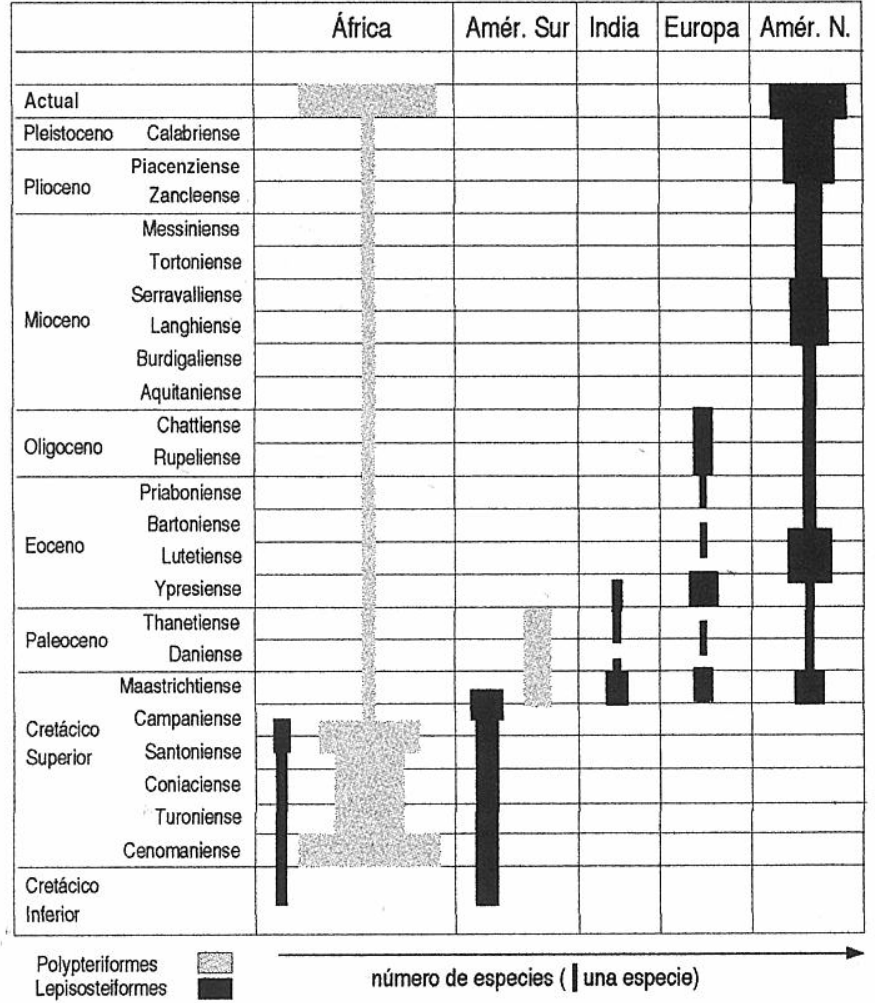

Figura 4. Comparación de las diversificaciones con su localización en los polipteriformes y los lepisosteiformes.

Comparison between polypteriform and lepisosteiform diversifications.

restringidas todas ellas a América del Norte). Al contrario, los polipteriformes no tuvieron la misma capacidad adaptativa para colonizar otros ambientes, a pesar de su diversificación más importante.

\section{CAUSAS INDUCTORAS DE LA DIVERSIFICACIÓN DE LOS POLIPTERIFORMES \\ Innovaciones clave}

Las pínulas de los polipteriformes pueden interpretarse como innovaciones clave porque constituyen un carácter de grupo monofilético de gran diversidad. Los actinopterigios tienen o bien pínulas (polipteriformes) o bien lepidotriquias articuladas (todos los otros grupos). Al contrario que todos los demás peces conocidos, el desarollo ontogenético de la pínula dorsal de los polipteriformes muestra una evolución muy particular con una aparición en una secuencia posteroanterior (Sewertzoff, 1924; Bartsch y Gemballa, 1992). No se conoce un patrón intermedio entre la formación de las pínulas con una secuencia postero-anterior y la contraria. El papel de esta innovación clave en la diversificación de los polipteriformes no es conocido. De hecho, los polipteriformes muestran algunas innovaciones clave sin fenotipos intermedios, como son la morfología vertebral característica, la estructura "contrachapada" de las escamas o las aletas pectorales braquiopterigianas. Sin embargo, la posibilidad de tolerar aguas sin oxígeno es 


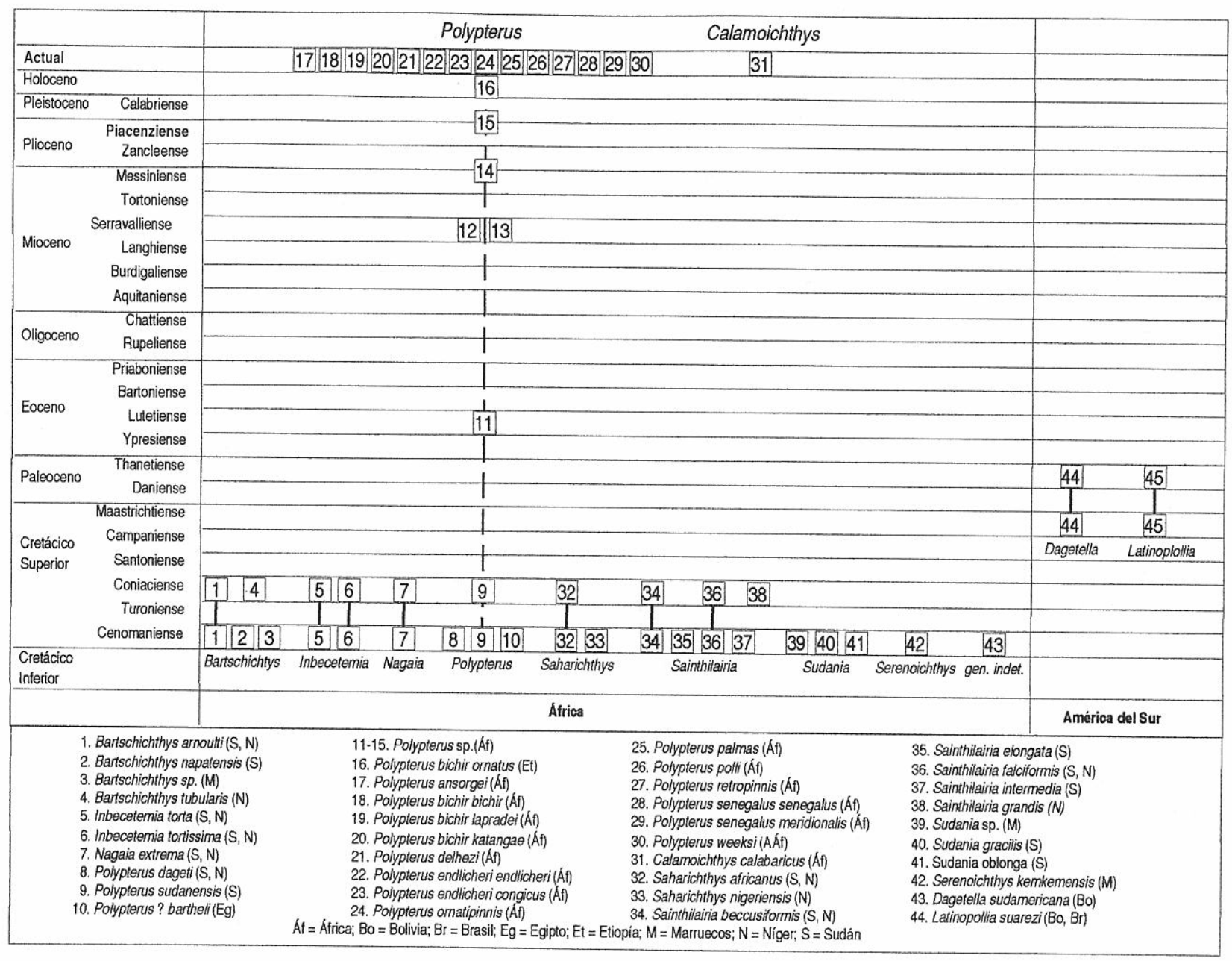

Figura 5. Diversificación de los polipteriformes desde el Cenomaniense. Polypteriform diversification from Cenomanian to Recent.

probablemente más importante para la diversificación. Si no son los únicos peces con esta posibilidad (Roberts, 1975), sí son los primeros junto a los Dipnoi que han desarrollado esta capacidad.

Las pínulas, innovaciones clave que no son previsibles, han aparecido súbitamente en un grupo monofilético de gran diversidad y tuvieron la capacidad de ser heredadas; no constituyen probablemente el motivo fundamental (o al menos, no el único) de la explosión súbita de los polipteriformes pero son elementos fosilizados que indican la diversidad del grupo.

Se puede pensar que la innovación de la respiración aérea fue una posibilidad de adaptación a diferentes ecosistemas, y consecuentemente de diversificación. Sin embargo, la misma innovación se conoce actualmente en otros grupos africanos de peces (Channidae, Lepidosirenidae, etc., Roberts, 1975) sin que pueda inducirse ninguna radiación súbita ni de la misma importancia.

\section{Aspectos paleoambientales}

Otros grupos, como las serpientes (Werner y Rage, 1994) y los cocodrilos (Werner, com. pers.), muestran también una diversificación explosiva en la misma época (Cenomaniense del Sudán) con su primera aparición. La paleotectónica durante el Cretácico se caracterizó en el Sudán por la reactivación de estructuras de grabens que originaron ambientes variados (Werner, 1998).

La última diversificación (sub-actual) de estos peces (Fig. 5) podría estar relacionada con el clima muy húmedo que se registra en África durante el Pleistoceno (Petit-Maire, 1983; Van Neer y Gayet, 1989). Sin embargo, un clima semejante en el Plioceno de América del Norte no ha provocado ninguna diversificación similar de los lepisosteidos (Miller, 1965).

De este modo, la diversificación de los polipteriformes, sin antecesores reconocidos, parece relacionada con las circunstancias externas (clima húmedo) y con la adquisición de una innovación clave. Esta innovación morfológica, con un papel no conocido, permite descubrir la diversificación pero no explica funcionalmente la radiación de los polipteriformes. Aquí también se carece aún de una explicación enteramente satisfactoria para la diversificación del grupo.

\section{CONCLUSIONES}

Para los dos ejemplos tan diferentes examinados, se admite que el registro fósil conocido facilita una imagen 
suficientemente fiel, y es una primera hipótesis que se puede sin duda impugnar pero eso supondría invalidar la mayor parte de los razonamientos paleontológicos. Admitiendo la validez de los datos, hemos buscado circunscribir los factores inductores potenciales, externos e intrínsecos. El conocimiento de todos los parámetros de los paleoambientes es probablemente casi siempre muy parcial. La búsqueda de innovaciones morfológicas clave se enfrenta con diferentes dificultades; algunas innovaciones no fosilizables requieren interpretaciones hipotéticas del material fósil (las filibranquias de los bivalvos); por otro lado, a veces no se puede decir si caracteres nuevos constituyen elementos adaptativos clave de la diversificación (las pínulas de los polipteriformes). Así, la mayoría de las veces, se carece de una explicación enteramente satisfactoria para la diversificación de un grupo. El estudio de las paleodiversificaciones tiene límites inherentes a la calidad del registro fósil; sin embargo, un desarrollo de las investigaciones incluyendo grupos numerosos y variados podrá, con un razonamiento inductivo, dar cuenta de los factores inductores y precisar las modalidades generales de los fenómenos.

Finalmente, a otro nivel más general de los mecanismos evolutivos, el estudio de estas paleodiversificaciones que muestran la rapidez de la aparición de los grupos observados plantea, otra vez, el problema del patrón darwiniano ya que no concuerda con el gradualismo.

\section{AGRADECIMIENTOS}

Expresamos nuestro agradecimiento al Dr. E. Villas por su ayuda en la revisión del idioma. Asimismo, agradecemos la lectura crítica al Dr. J. Truyols.

\section{BIBLIOGRAFÍA}

Babin, C. 1993. Rôle des plates-formes gondwaniennes dans les diversifications des mollusques bivalves durant l'Ordovicien. Bulletin de la Société géologique de France, 164, 141-153.

Babin, C. 1995. The initial Ordovician bivalve mollusc radiations on the western Gondwanan shelves. In: Ordovician Odyssey (Eds. J.D. Cooper, M.L. Droser \& S.C. Finney), Pacific Section Society of Sedimentary Geology, 77, 491-498.

Babin, C. and Guttiérez-Marco , J.C. 1991. Middle Ordovician bivalves from Spain and their phyletic and palaeogeographic significance. Palaeontology, 34, 109147.

Bartsch, P. and Gemballa, S. 1992. On the anatomy and development of the vertebral column and pterygiophores in Polypterus senegalus Cuvier, 1829 ("Pisces", Polypteriformes). Zoologische Jahrbuch Anatomie, 122, 497-529.

Cope, J.C.W. 1995. The early evolution of the Bivalvia. In: Origin and evolutionary radiation of the Mollusca (Ed.
J.D.Taylor). Oxford University Press, 361-370 (1996).

Cope, J.C.W. 1996. Early Ordovician (Arenig) bivalves from the Llangynog Inlier, South Wales, Palaeontology, 39, 979-1025.

Cope, J.C.W. 1997. The early phylogeny of the class Bivalvia. Palaeontology, 40, 713-746.

Cope, J.C.W. and Babin, C. 1999. Diversification of bivalves in the Ordovician. Geobios, 32, 175-185.

Dutheil, D.B. 1999. The first articulated fossil cladistian: Serenoichthys kemkemensis, gen. et sp. nov. (Actinopterygii: Cladistia), from the late Cretaceous of Morocco. Journal of Vertebrate Paleontology, 19, $243-$ 246.

Gardiner, B.C. 1984. The relationships of the palaeoniscid fishes, a review based on new specimens of Mimia and Moythomasia from the Upper Devonian of Western Australia. Bulletin of the British Museum (Natural History), Geological series, 37 (4), 175-428.

Gayet, M. and Meunier, F.J. 1991. First discovery of Polypteridae (Pisces, Cladistia, Polypteriformes) outside of Africa. Geobios, 24, 463-466.

Gayet, M. et Meunier, F.J. 1992. Polypteriformes du Maastrichtien et du Paléocène de Bolivie. Geobios, m. sp. 14, 159-168.

Gayet, M. et Meunier, F.J. 1996. Nouveaux Polyptériformes du gisement coniacien-santonien d'In Becetem (Niger). Comptes Rendus Académie des Sciences de Paris, 322, IIa, 701-707.

Gayet, M. and Meunier, F.J. 1998. Maastrichtian to Early Late Paleocene freshwater Osteichthyes of Bolivia: additions and comments. In: Phylogeny and classification of neotropical fish (Eds. L.R. Malabarba, R.E. Reis, R.P. Vari, Z.M. Lucena \& C.A.S. Lucena), Porto Alegre Edipucrs, 85-110.

Gayet, M., Meunier, F.J. and Werner, C. 1997. Strange Polypteriformes from the Upper Cretaceous of In Becetem (Niger) and Wadi Milk Formation (Sudan). Geobios, m. sp. 20, 249-255.

Gayet, M., Meunier, F.J. and Werner, C. 1998. Difference in diversification of some Actinopterygii: Polypteriformes and Lepisosteiformes. In: Symposium Paléodiversifications: terres et mers comparées, Lyon 6-8 juillet 1998, Résumés, 23.

Gayet, M., Meunier, F.J. and Werner, C. (en prensa). Difference in diversification of some Actinopterygii: Polypteriformes and Lepisosteiformes. Palaeontology.

Geyer G. and Strend M. 1998. Middle Cambrian pelecypods from the Anti-Atlas, Morocco. Revista Española de Paleontología, N $^{\mathbf{0}}$ extr., 83-96.

Harper, J.L. and Hawksworth, D.L. 1994. Biodiversity: measurement and estimation. Preface. Philosophical Transactions of the Royal Society of London B, 345, 512.

Hinz-Schallreuter, I. 1995. Muscheln (Pelecypoda) aus dem Mittelkambrium von Bornholm. Geschiebekunde aktuell, 11, 71-84.

Meunier, F.J. and Gayet, M. 1996. A new polypteriform from the Late Cretaceous and the Middle Paleocene of South America. In: Mesozoic Fishes - Systematics and Paleoecology (Eds. G. Arratia \& G. Viohl), Verlag, Dr. 
F. Pfeil, München, 95-105.

Miller, R.R. 1965. Quaternary freshwater fishes of North America. In: The Quaternary of the U.S. (Eds. H.E. Jr Wright. \& G.D. Frey). International Association on Quaternary Research. Princeton University Press, 569581.

Myers, G.S. 1938. Fresh-water fishes and west Indian zoogeography. Smithsonian Report 1937, 339-364.

Myers, G.S. 1949. Salt-tolerance of fresh-water fish groups in relation to zoogeographical problems. Bijdragen tot de Dierkunde, Amsterdam, 28, 315-322.

Petit-Maire, N. 1983. Nouvelles données sur les paléoenvironnements holocènes de l'Afrique de l'Ouest. In: Sahara ou Sahel? Quaternaire récent du Bassin de Taoudenni (Mali) (Eds. N. Petit-Maire \& J. Riser), Université de Marseille-Luminy, France, 413-418.

Roberts, T.R. 1975. Geographical distribution of African freshwater fishes. Zoological Journal of the Linnean Society of London, 57, 249-319.

Schaal, S. 1984. Oberkretazische Osteichthyes (Knochenfische) aus dem Bereich von Bahariya und Kharga, Ägypten, und ihre Aussagen zur Palökologie und Stratigraphie. Berliner geowissenchaftliche Abhandlungen, A 53,1-79.

Sereno, P.C., Dutheil D.B., Iarochene, M., Larsson, H.C.E.,
Lyon, G.H., Magwene, P.M., Sidor, C.A., Varrichio, D.J., and Wilson, J.A. 1996. Predatory dinosaurs from the Sahara and late Cretaceous faunal differenciation. Science, 272, 986-991.

Sewertzoff, A.N. 1924. The development of the dorsal fin of Polypterus delhesi. Journal of Morphology, 38, 551-580.

Van Neer, W. et Gayet, M. 1989. Etude des poissons en provenance des sites holocènes du bassin de TaoudenniAraouane (Mali). Bulletin du Muséum national d'Histoire naturelle de Paris, 4e série, 10, sect. C (4), 343-383.

Werner, C. 1994. Die kontinentale Wirbeltierfauna aus der unteren Oberkreide des Sudan (Wadi Milk Formation). Berliner geowissenchaftliche Abhandlungen E 13, 221249.

Werner, C. 1998. Palaeodiversity of the Cenomanian vertebrate fauna of Sudan. Paléodiversifications, terres et mers comparées, Lyon, 6-8 juillet 1998, Résumés, 35.

Werner, C. and Gayet, M. 1997. New fossil Polypterids from the Cenomanian of Sudan. An evidence of their high diversity in the early Late Cretaceous. Cybium, 6, 67-81.

Werner, C. and Rage, J.-Cl. 1994. Mid-Cretaceous snakes from Sudan. A preliminary report on an unexpectedly diverse snake fauna. Comptes Rendus de l'Académie des Sciences de Paris, 319, II, 247-252. 